\title{
Research on the control and algorithm of location for electro-hydraulic servo lording system
}

\author{
QIANG Yi ${ }^{11, a}$, SUI Yan ${ }^{1, b}$, ZHANG Jun-lin ${ }^{2, c}$, MA Lei, ${ }^{1, d}$ \\ ${ }^{1}$ School of Civil Engineering, Xi'an Univ. of Arch \& Tech, China \\ ${ }^{2}$ School of Information and Control Engineering, Xi'an Univ. of Arch \& Tech, China \\ a13700284274@126.com, bsuiyanmyy@163.com, ${ }^{\mathrm{a}}$ zhangjl9966@163.com, ${ }^{\mathrm{d}}$ malei0504@126.com
}

Keywords: Servo hydraulic system; Fuzzy control; Multi-modal control

\begin{abstract}
Based on the seismic lab platform In this paper,aimed at model parameters of electro-hydraulic servo loading system,position has the characteristic of variability and susceptible to external interference,the combination of fuzzy control and PID control,we propose a multi-modal(P-Fuzzy adaptive PID-PI)control strategy of segmentation. This control method using fast response characteristic of $\mathrm{P}$ control,adaptive characteristic of Fuzzy adaptive PID system modal,as well as steady-state PI control system quickly adjust the characteristics of dynamic variation to achieve the position of electro-hydraulic servo loading system stability and regulation. Through simulation analysis concluded that to achieve well control performance,with important theoretical and practical value.
\end{abstract}

\section{Introduction}

Large-scale civil engineering structures seismic experiments (including pseudo-dynamic test and multi-point quasi-static loading test) must have the hardware test electro-hydraulic servo control system and load support [1], but due to site conditions, oil temperature and oil viscosity impact and other uncertainties, electro-hydraulic servo loading system in the electro-hydraulic servo valve has a greater volatility and poor immunity characteristics of the hydraulic cylinder position control, the use of existing PID control can not meet the hydraulic Servo Loading System control requirements, and therefore needs to be improved to its control [2].

\section{Location model building}

Electro-hydraulic servo loading system consists of two parts, electrical and hydraulic components. The hydraulic part is a liquid (including an oil, a high water-based hydraulic oil or synthetic liquid) as a medium, the pressure generated by the pressure fluid depend on the amount of mechanical energy to achieve a variety of (force, displacement, or velocity, etc.) is transmitted. A typical electro-hydraulic servo loading system shown in Figure 1 [3]:

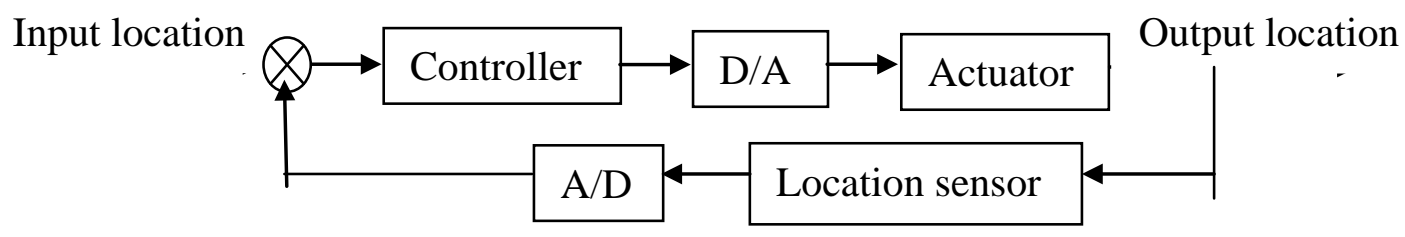

Fig1 A typical block diagram of the electro-hydraulic servo loading system

How it works: After the command input voltage and the feedback voltage measurement sensors provide feedback compared to get the error, after the error by the amplifier, converted to control the current electro-hydraulic servo valve spool displacement control current electro-hydraulic servo valve proportional, by controlling the direction and size of the valve opening to control the flow of

Fund Project: National Natural Science Foundation of major research projects (51178382); Key Laboratory of Science and Technology Foundation of Western architecture independent research (K01338); Xi’an University of Architecture \& Technology Youth Fund(DA01141) 
oil into the hydraulic cylinder and flow, by a hydraulic actuator push rod moves in the direction of the load together reduce errors. This constitutes the electro-hydraulic servo feedback control system. By substituting the data obtained for the input spool displacement between XV and the output Y of the transfer function of the piston position:

$$
G(s)=\frac{3275}{0.05225 s^{3}+0.47 s^{2}+s}
$$

This formula (1) electro-hydraulic servo valve controlled hydraulic cylinder transfer function for the simulation model of the system [4].

\section{Control algorithm}

When a deviation greater than a certain threshold, with proportional control, in order to improve the response speed of the system, speeding up the response process; when a small deviation below the threshold into another larger, switch to FPID controlled to provide the system damping performance, reduce overshoot response process. This combines the advantages of proportional control and fuzzy control. However, since there is no control FPID integral part, but the amount of processing of the input is discrete and limited, i.e., the control plane is stepped rather than smooth, small amplitude oscillations may occur in the vicinity of the equilibrium point. The PI control in a small area near the balance point adjustment effect is ideal, and its integral role may eventually eliminate static bias. System control block diagram shown in Figure 2 [5].

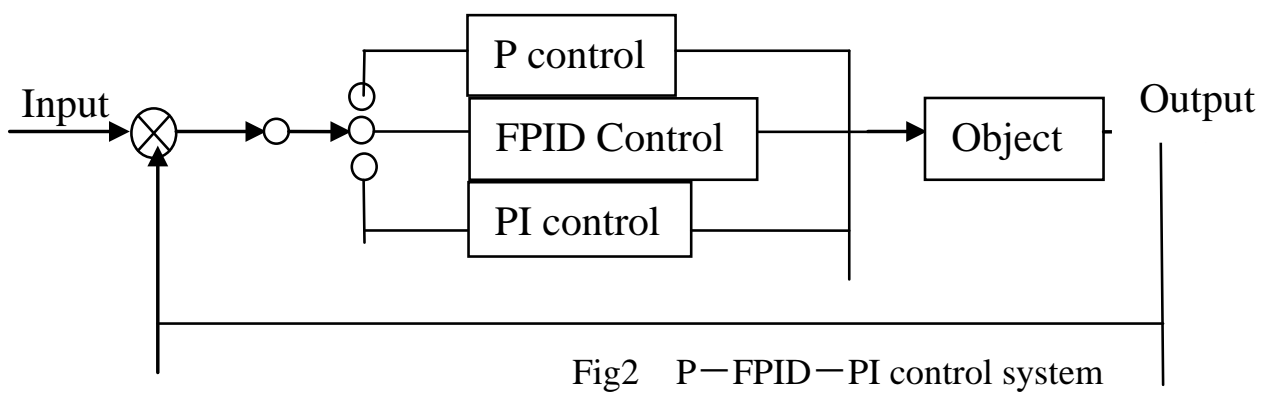

\section{Control Simulation}

The main advantage of Matlab fuzzy logic toolbox article (Fuzzy Logic Toolbox) graphical user interface (GUI) to establish fuzzy inference system, and simulation analysis. The GUI toolkit has five tools: Fuzzy Inference System (Fuzzy Inference System, FIS) editor, the editor of membership functions, fuzzy rule editor; 2 viewer, that fuzzy rules to observe and output surface observer. These graphical tools are dynamically linked to each other, very user-friendly fuzzy inference system debugging. Using System P-FPID-PI control simulation diagram shown in Figure 3. 


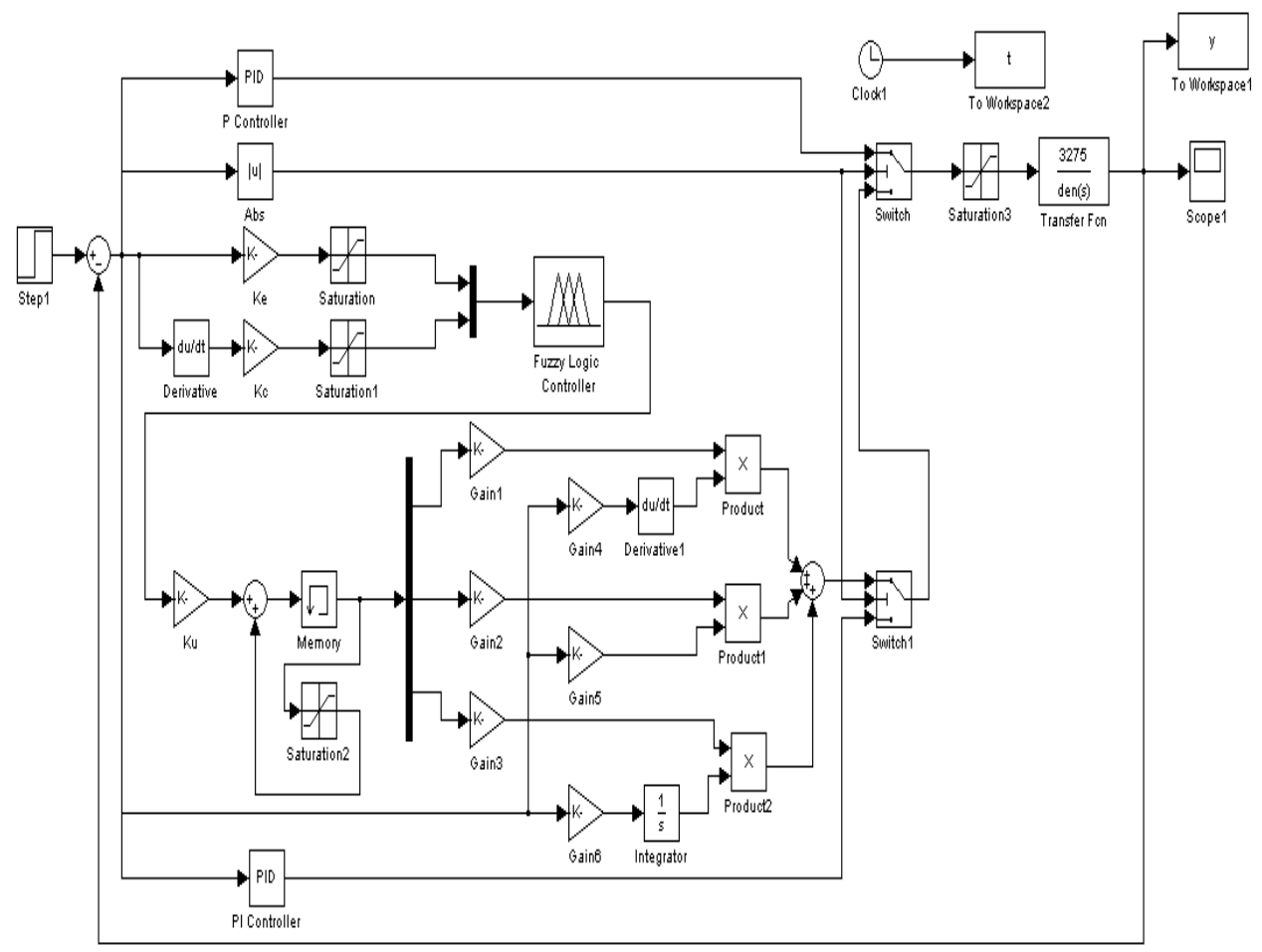

Fig3 P-FPID - PI control simulation diagram

The PID control, FPID control and P-FPID-PI control simulation graph step signal into a triangular wave signal amplitude, Figure 4 shows a triangular wave after adding PID control, FPID control and P-FPID-PI control simulation comparison graph.

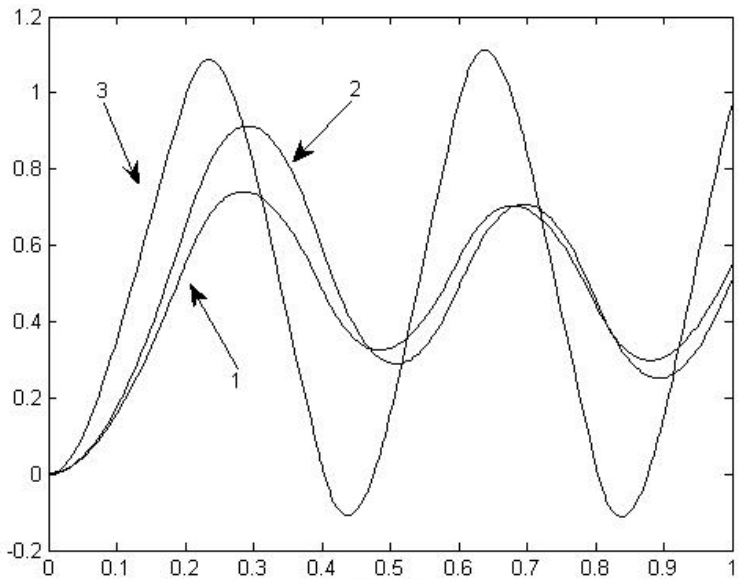

Fig4 PID、FPID and P-FPID-PI control simulation graph after a triangular wave

In Figure 4, vertical axis is location and horizontal axis is time,curve 1 for P-FPID-PI control simulation curve, 2 FPID control simulation curve, curve 3 for PID control simulation curve, by comparing the three curves in the figure can be seen, P-FPID-PI control compared with conventional PID control, which improves the stability of the system, reducing the overshoot, improved dynamic characteristics of the system; FPID compared with control, it improves the system stability, reduced overshoot on the basis of to improve the response time of the equilibrium point.

\section{Conclusion}

This paper analyzes the PID control and fuzzy control methods to control these two basic principles of electro-hydraulic servo loading system for MTS and time-varying characteristics of poor immunity, the fuzzy control and conventional PID control combined with the design of a P-FPID-PI control algorithm, and the algorithm is explained control principle, the use of MATLAB Simulink 
tools to build a simulation model of the system, the control system simulation objects. Simulation results show that: P-FPID-PI control with a strong fast, interference, the system has strong adaptability.

\section{Reference}

[1]GUO Xin,BAI Guo-liang,LIU Xu,ZHU Li-hua. Implementation of data collection simultaneously in seismic PSD test[J]. J. of Xi' an University of Architecture \& Technology, 2012, 474-479.

[2]YU Bing,PENG You-duo,LIU Fan-mao. Fuzzy Control and its Application in Hydraulic Servo System[J]. Hydraulic and pneumatic,2006,56-63.

[3]XUE Zhao-hong,CHI Yi-lin,LIU Dao-yu. Application of Three-Dimensional Fuzzy Control in Electro-hydraulic Position Servo Control System[J].Hydraulic and pneumatic,2005,45-47.

[4]LIU Xing-tang,WU Xiao-yan. Modern systems modeling and simulation technology[M].Xi'an: Northwestern Polytechnical University,2001

[5]ZHANG Tao,XUE Peng-jian,JIANG Jing-ping. Position Follow UP System Based on Multi State Control Method by Fuzzy PID Control[J].Jiangnan University,2008,7(3):304-307. 